\title{
From cord to caudate: characterizing umbilical cord blood stem cells and their paracrine interactions with the injured brain
}

\author{
Priya F. Maillacheruvu' ${ }^{1}$, Lauren M. Engel ${ }^{1}$, Isaiah T. Crum ${ }^{1}$, Devendra K. Agrawal ${ }^{2}$ and Eric S. Peeples ${ }^{1}$
}

Stem cells are proving to be a promising therapy for a wide range of pediatric disorders, from neonatal hypoxic-ischemic encephalopathy to pediatric leukemia. Owing to their low immunogenicity and ease of availability, umbilical cord blood (UCB) progenitor cells are increasingly replacing fetal- and adult-derived cells in therapeutic settings. Multiple environmental and demographic factors affect the number and type of stem cells extracted from UCB, and these differences have been associated with disparities in outcomes after transplantation. To avoid variations in efficacy, as well as the potential adverse effects of stem cell transplantation, evaluation of the stem cell secretome is critical to identify key paracrine signals released by the stem cells that could be used to provide similar neuroprotective effects to stem cell transplantation. This article describes the cell types found in UCB and reviews the available literature surrounding the effects of collection timing and volume, maternal risk factors, delivery characteristics, and neonatal demographics on the cellular composition of UCB. In addition, the current findings regarding the stem cell secretome are discussed to identify factors that could be used to supplement or replace stem cell transplantation in pediatric neuroprotection.

$\mathbf{S}$ tem cells have demonstrated significant promise in preclinical studies of neuronal repair in pediatric disorders such as hypoxic-ischemic encephalopathy (HIE), stroke, intraventricular hemorrhage, and traumatic brain injury $(1,2)$. In addition, early clinical studies have shown both clinical feasibility for administration of stem cells after neonatal HIE and efficacy in decreasing cerebral inflammation in pediatric patients with cerebral palsy $(3,4)$. Umbilical cord blood (UCB) is an attractive source of stem cells, since its collection is noninvasive, painless, and does not evoke the ethical concerns of embryonic stem cells. Although the number of nucleated cells recovered from UCB is significantly lower than that from bone marrow $(\mathrm{BM})$, there is a higher frequency of primitive cells in UCB. The immature T-lymphocytes contained in UCB suppress the activation of lymphocytes and natural killer cells, resulting in low immunogenicity and pathogenicity $(5,6)$. The ease of collection combined with the increased proliferative capacity of the more primitive cells compensate for the potential difficulty obtaining adequate numbers of nucleated cells, supporting the use of UCB as a valuable source of stem cells for therapeutic interventions.

To attempt to overcome the paucity of nucleated cells in $\mathrm{UCB}$, one must understand not only the conditions that affect the numbers and quality of stem cells in UCB but also the neurotrophic and growth factors involved in stem cellmediated repair that can potentially be upregulated through genetic manipulation of the cells. These factors can enhance the efficacy of stem cells if delivered together or may act as a replacement for cell transplantation. This review will discuss the conditions affecting UCB stem cell numbers and quality, as well as the stem cell secretome and its interactions with the injured brain.

\section{STEM CELL TYPES DERIVED FROM THE UMBILICAL CORD AND PLACENTA}

UCB is not the only source of UC-derived stem cells; other UC-derived populations such as UC perivascular cells, umbilical vein endothelial cells, and UC-lining stem cells have also been suggested as alternatives to UCB (Figure 1). Compared with UCB, higher numbers of mesenchymal stromal/stem cells (MSCs) can reliably be extracted from the various compartments of the UC. One of the most readily accessible UC stem cell sources is the Wharton's jelly. Wharton's jelly is the mucous connective tissue between the amnion and the umbilical vessels and contains a native population of MSCs referred to as Wharton's jelly stem cells (7). Wharton's jelly stem cells demonstrate a higher frequency of colony-forming unit fibroblasts than either UCB MSCs or BM MSCs (8), and like UCB MSCs, Wharton's jelly stem cell MSCs are multipotent (9). In addition to UC tissue, epithelial and mesenchymal cell types may also be derived from the amnion and chorion of the placenta (10). This review will focus primarily on UCB, as most of the UC-derived stem cell literature to date has focused primarily on UCB. It is important, however, to understand that other stem cell populations extracted from the UC and placenta are also

${ }^{1}$ Department of Pediatrics, Children's Hospital and Medical Center, University of Nebraska Medical Center, Omaha, NE; ${ }^{2}$ Department of Clinical and Translational Science, Creighton University School of Medicine, Omaha, NE. Correspondence: Eric S. Peeples (eric.peeples@unmc.edu)

Received 17 May 2017; accepted 2 October 2017; advance online publication 1 November 2017. doi:10.1038/pr.2017.251 


\section{Review | Maillacheruvu et al.}

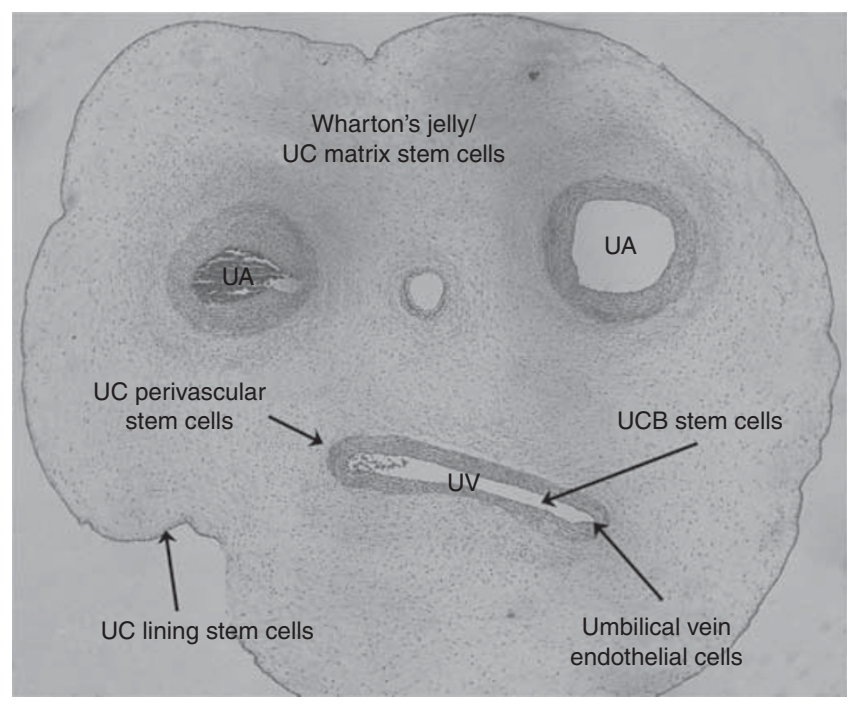

Figure 1. Sections of the umbilical cord that may be harvested for stem cells. UA, umbilical artery; UC, umbilical cord; UCB, umbilical cord blood; UV, umbilical vein. Figure adapted and modified from (ref. 97) (c) 2016 Uthman CC-BY, version 2.0.

being examined for their potential therapeutic effect in pediatric brain injury, and we may discover that UCB MSCs are less effective than one or more of the other UC-derived populations.

UCB contains numerous stem and progenitor cell types, including hematopoietic stem cells, MSCs, and the unipotent endothelial progenitor cells (Figure 2). MSCs, however, are the only UCB-derived stem cell population with the potential for neuronal differentiation, and as such, they will be the primary focus of this review. MSCs are multipotent nonhematopoietic cells defined by the International Society for Cellular Therapy as having $\geq 95 \%$ expression of CD105, CD73, and CD90, and $\leq 2 \%$ expression of CD45, CD34, CD14, CD11b, CD79a, or CD19, and HLA-DR surface molecules. Additionally, an MSC must be able to differentiate into osteoblasts, chondroblasts, and adipocytes in vitro (11).

A unique subset of MSCs has been identified in UCB, which express pluripotent stem cell markers and are capable of differentiating into neurons after exposure to simple induction conditions in vitro (12). Other UCB MSCs have been induced in vitro to express neural-specific antigens and take on the morphology of neural cells after being exposed to neural culture (13). In support of this differential capability, some studies have reported migration and differentiation of UCB mononuclear cells into astrocytes when transplanted either intraperitoneally or intracerebrally in a rodent model of neonatal hypoxic-ischemic brain injury (14-16). Compared with BM or UC cells, UCB contains lower number of MSCs (17,18), making it increasingly important to understand the factors that may increase the numbers or potency of MSCs in UCB to maximize their therapeutic potential.

\section{EFFECT OF COLLECTION FACTORS ON UCB}

Collection factors such as increased blood volume and decreased time to isolation are two of the primary drivers in successful isolation of stem cells from UCB (Table 1). AlQahtani et al. (19) demonstrated that collection volume was the most significant factor among 16 maternal, neonatal, and obstetric variables in predicting the number of total nucleated cells (TNCs) derived from UCB samples (19). The few studies that have assessed these factors in greater detail suggest that a net volume $>33-80 \mathrm{ml}$ and time from collection to isolation $<10-15 \mathrm{~h}$ significantly increase the yield of CD34+ cells from UCB $(20,21)$. Because of the strong association between cord blood volume and stem cell yield, the increasing frequency of delayed cord clamping (DCC) has been closely analyzed due to the concern that it could decrease the volume of blood remaining in the placenta and UCB.

Two studies evaluating the effects of DCC on UCB characteristics have found lower TNC counts in DCC compared with immediate cord clamping, with $17-37 \%$ of samples meeting a TNC threshold of $15 \times 10^{8}$ cells for eligibility for cord banking in the DCC group and $37-47 \%$ in the immediate cord clamping group $(22,23)$. The difference was no longer significant at a threshold of $12.5 \times 10^{8}$ cells, however, with $52 \%$ of the DCC group vs. $60 \%$ in the immediate cord clamping group meeting criteria (23). Despite the significant difference between groups, the authors highlighted that many cord blood units still met the highest threshold despite DCC, implying that collection of quality UCB stem cells continues to be feasible despite DCC. In addition, the clinical benefit of DCC likely outweighs the potential for lower stem cell yield. Not only has DCC been associated with decreases in intraventricular hemorrhage and respiratory distress syndrome, but a number of authors have argued that DCC may be the most effective way to noninvasively administer autologous stem cells (24-26).

Cord milking is another procedure that has been used to improve passage of placental blood to the neonate immediately after birth, especially in situations where DCC may be inappropriate due to the need for resuscitation (27). Although one study demonstrated higher systemic blood flow after cord milking compared with DCC in preterm infants (28), there does not appear to be a significant difference in the amount of blood transferred between the two procedures (29), and the effect of cord milking on UCB collection has not yet been thoroughly evaluated.

\section{EFFECT OF FETAL STRESS ON UCB}

When exposed to certain environmental factors such as acute hypoxia, stem cells demonstrate improved proliferation and function. UCB MSCs cultured under hypoxic conditions display increased hypoxia-inducible factor- $1 \alpha$ expression, angiogenic cytokines, and improved proliferative capacity in vitro. Transplantation of hypoxia-preconditioned stem cells into a mouse model of an ischemic limb resulted in decreased muscle atrophy, bone loss, and apoptosis, and increased 


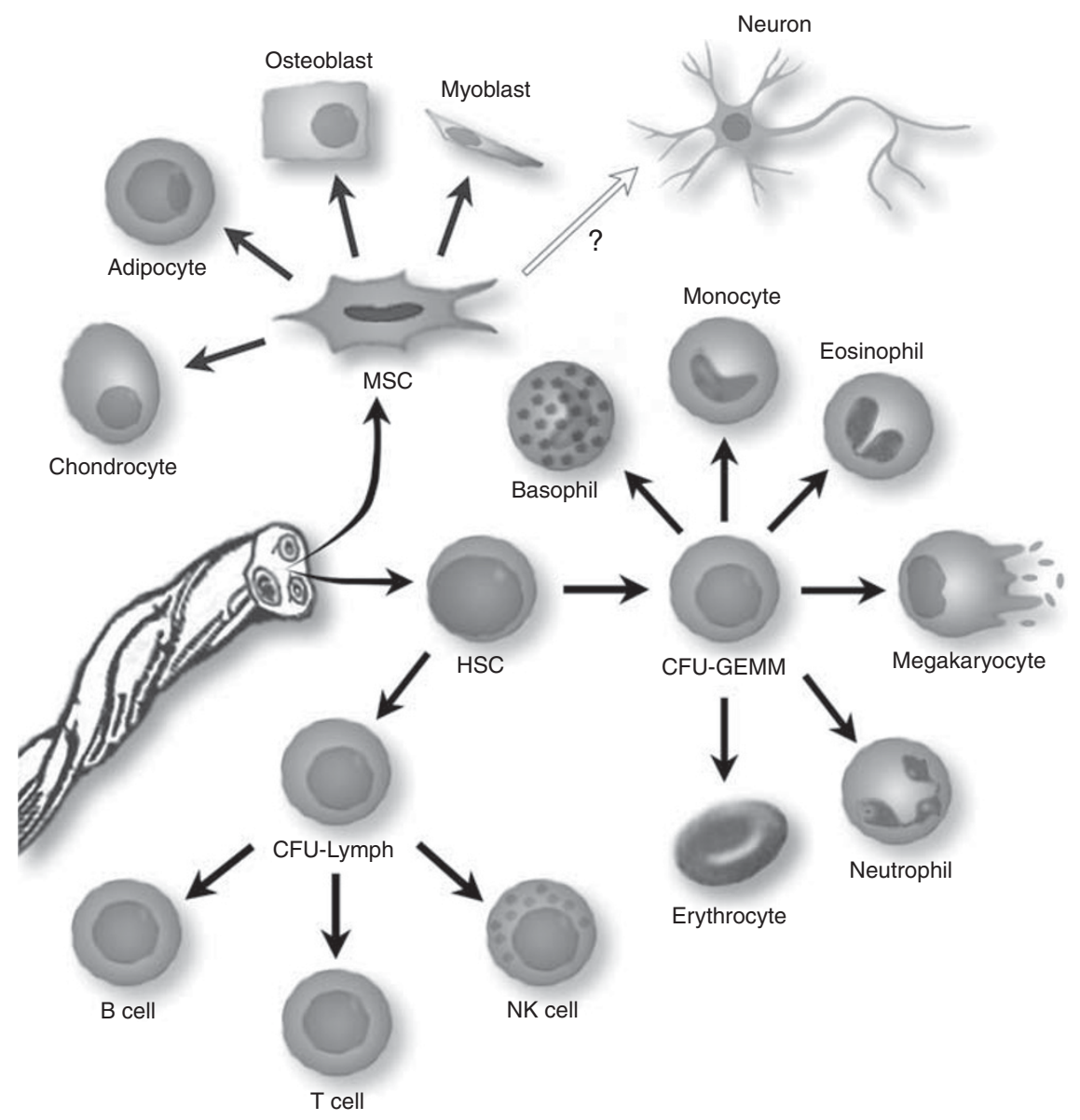

Figure 2. Potential differentiation of mesenchymal (MSC) and hematopoietic stem cells (HSC) derived from the umbilical cord and umbilical cord blood. CFU, colony-forming unit; GEMM, granulocyte, erythroid, macrophage, and megakaryocyte.

Table 1. Summary of the effects of maternal and neonatal factors on umbilical cord blood characteristics

\begin{tabular}{|c|c|c|c|c|c|c|c|c|c|c|c|}
\hline & \multicolumn{3}{|c|}{ Maternal factors } & \multicolumn{8}{|c|}{ Neonatal factors } \\
\hline & \multirow[t]{2}{*}{ Weight } & \multirow[t]{2}{*}{ Parity } & \multirow[t]{2}{*}{ Age } & \multirow[t]{2}{*}{ BW } & \multirow[t]{2}{*}{ GA } & \multirow[t]{2}{*}{ Placental weight } & \multirow[t]{2}{*}{ UC length } & \multicolumn{2}{|c|}{ Sex } & \multicolumn{2}{|c|}{ Race $^{a}$} \\
\hline & & & & & & & & M & $\mathrm{F}$ & Caucasian & Black \\
\hline CFU & & - & & ++ & - & + & + & ++ & + & + & \\
\hline CD34+ & & - & & ++ & -- & + & + & ++ & + & + & \\
\hline
\end{tabular}

Abbreviations: BW, birth weight; CFU, colony-forming units; GA, gestational age; TNC, total nucleated cells; UC, umbilical cord; UCB, umbilical cord blood.

Empty cells represent associations without adequate data.

a Listed effects representative of listed race vs. other races. Other races were not included due to inadequate data.

capillary and arteriole density correlating with the level of hypoxic preconditioning $(30,31)$.

The effects of hypoxia on MSC function have not been well evaluated clinically. Studies of UCB endothelial colonyforming cells (ECFC), however, have assessed the effects of chronic hypoxia by investigating fetuses exposed to placental insufficiency caused by maternal diabetes mellitus or preeclampsia. As opposed to the improved proliferation and function of MSCs after acute in vitro hypoxia, UCB ECFC experiencing chronic hypoxia secondary to maternal diabetes tend to exhibit decreased vessel formation, increased senescence, and decreased proliferation, especially after multiple passages $(32,33)$. This may be in part due to a difference in the ability to express vascular endothelial growth factor. UCB ECFCs obtained from patients from nondiabetic pregnancies demonstrated increased vascular endothelial growth factor-A expression when exposed in vitro to moderate hypoxia for $72 \mathrm{~h}$. ECFCs obtained from pregnancies affected by gestational diabetes showed no increase in vascular endothelial growth factor-A expression after hypoxia (34). In mothers with preeclampsia, the UCB volume, TNC count, CD34+ cell count, and the number of ECFCs in UCB are 


\section{Review | Maillacheruvu et al.}

significantly decreased, and the cells are slower to emerge in culture compared with controls $(35,36)$. Taken together, these findings suggest that controlled acute hypoxia may help to increase the angiogenic function of UCB stem cells in vitro, although conditions such as gestational diabetes or preeclampsia that may cause chronic hypoxia in vivo tend to result in decreased ECFC angiogenic function.

\section{EFFECT OF MATERNAL FACTORS ON UCB}

In addition to maternal diseases such as preeclampsia and diabetes mellitus, other pregnancy complications and lifestyle choices can influence the quality and quantity of cord blood. For instance, although preeclampsia has an overall negative effect on the volume and cell count of UCB, maternal hypertension without preeclampsia has been shown to produce UCB with higher CD34+ cell counts (37). Maternal smoking has no documented effect on the quality of UCB, but has been demonstrated to result in lower UCB volume than in nonsmoking pregnancies (38). It is unclear if this is due directly to smoking or is secondary to smoking's effects on fetal growth, resulting in lower birth weight.

Other maternal factors such as maternal body habitus and parity have also been associated with cord blood quality. Higher maternal weight is associated with an increased TNC count $(37,39)$, although no association was found with maternal height. In addition, lower parity is positively correlated with TNC, colony-forming units (CFU), and CD34+ counts $(39,40)$, and with each additional previous birth there is a $17 \%$ decrease in CD34+ counts (38). The influence of other factors such as maternal age is still poorly understood. Although some studies have found that younger maternal age leads to higher cell counts in UCB, others have found that mothers 20 years or older have higher CFU, CD34+, and TNC counts $(21,41)$. Still, more studies have found no correlation between maternal age and quality of UCB (38).

\section{EFFECT OF NEONATAL FACTORS ON UCB}

The quantity and quality of UCB correlate with several neonatal factors, including birth weight, placental weight, and umbilical cord length. Studies have consistently demonstrated an association between higher birth weight and improved UCB quantity and quality, defined as a higher number of CFU, CD34+ cells, or TNC $(21,38,42)$. It has been estimated that every $500 \mathrm{~g}$ increase in birthweight results in a $6 \%$ higher volume of UCB collected. The same increase in birthweight increases the CFU yield by $9-21 \%$, CD $34+$ count by $11-28 \%$, TNC count by $6-11 \%$, and the ability to produce an acceptable UCB TNC count of $8 \times 10^{8}$ cells by $40 \%$ $(21,38,43)$. In a multivariate model assessing an association between TNC count and 10 different maternal and neonatal factors, birth weight was the strongest predictor of TNC count (39). In addition to birth weight, placental weight and umbilical cord length are also positively correlated with the volume of UCB collected and the number of TNC, CD34+ cells, and CFU $(40,41)$.
Longer gestation is correlated with a higher volume of UCB extracted and a higher TNC count. The cells extracted, however, tend to be more mature at higher gestational ages, demonstrating less CD34+ cells and lower CFU (39). For each additional week of gestational age, TNC count increases by $3 \%$ and $\mathrm{CD} 34+$ counts decrease by $9 \%$ (38). The increase in TNC count is thought to be due in part to increased placental size and weight at higher gestational ages. This balance between quantity and quality of UCB is important when considering the therapeutic potential of UCB. Preterm UCB contains fewer nucleated cells, but those that are extracted have increased proliferative capacity compared with term and post-term neonates $(21,44)$. Conversely, term and post-term UCB contains significantly more nucleated cells, resulting in $46 \%$ more successful cord blood units collected, but the cells contained in the units are less potent $(21,42)$.

The sex of the neonate may also influence the quantity and quality of the UCB obtained. Males tend to have higher cord blood volume, $\mathrm{CFU}$, and $\mathrm{CD} 34+$ counts, whereas females have a higher TNC count $(21,38,45-47)$. It is important to note, however, that many neonatal variables are highly correlated and may confound any observed univariate associations. For instance, there is a strong correlation between sex and birth weight, with male infants having higher birthweights on average than females. Thus, it is unclear if the relationship between sex and UCB characteristics is a true correlation or primarily due to the difference in birth weight. After correcting for birth weight, one study found that males have higher $\mathrm{CD} 34+$ count, $\mathrm{CFU}$, and hematopoietic progenitor cell concentration (46), although another found that there was no association between sex and UCB quality (43). Further studies assessing the association between neonatal sex and UCB characteristics should include multivariate analyses to correct for birth weight.

One benefit of the ease of UCB collection is that it allows for increased potential for collection from underrepresented races and ethnicities; however, race may also influence the quality of the UCB collected. The effect of race on UCB quantity is controversial, as some studies have found similar collection volumes between all races, and others have found decreased volumes in African-American infants $(40,48)$. Regardless of the volume, Caucasian neonates have been found to have the highest $\mathrm{CD} 34+$ counts and CFU, followed by multiracial, Hispanic, African American, and Asian neonates $(21,45)$. Some studies have also found higher TNC counts in Caucasians, although others have found no significant difference between races $(21,38,42)$. One of the greatest limitations to successfully answering the question of whether there is an association between race and UCB is that the current literature does not accurately represent the racial diversity present in most communities. Most studies rely on self-reported racial and ethnic demographics that often do not account for the race or ethnicity of the father, and almost universally do not account for multiracial backgrounds. Future studies using more detailed racial data or genetic 
analyses will be necessary to truly understand the effects of race on the quality of $\mathrm{UCB}$.

\section{EFFECT OF DELIVERY FACTORS ON UCB}

Although many studies have attempted to assess the relationship between mode of delivery and UCB, much of the data have been conflicting. One of the few consistent findings is that there tends to be a higher volume of UCB collected in cesarean deliveries vs. vaginal deliveries. The difference in volume may reflect the active manipulation of the placenta during a C-section as opposed to physiologic delivery of the placenta in vaginal deliveries, or potentially the difference in infant position; most vaginal deliveries result in the infant positioned lower than the placenta, allowing gravity to facilitate blood transfer from the placenta to the infant $(41,49)$. Last, the controlled nature of the operating room may be why UCB collection has been found to be $\sim 43 \%$ more successful after C-section compared with vaginal deliveries $(42,43)$.

Similar inconsistencies have come from studies assessing TNC counts between modes of delivery. Some studies have demonstrated higher TNC counts and cell quality in vaginal deliveries, which has been cited as being due to the stress on the fetus that results from labor $(37,48)$. Supporting that theory, C-section deliveries were found in one study to be $40 \%$ less likely than vaginal deliveries to have adequate TNC counts for clinical use $(42,43)$. Several other studies, however, have demonstrated higher TNC counts in C-section deliveries, hypothesizing that the more rapid UC clamp time in Cesarean deliveries results in more blood in the placenta and umbilical cord, and therefore increased TNCs $(42,49)$. A similar controversy exists regarding CFU and CD34+ cells, with some studies demonstrating higher total CFU or CD34+ cells in cesarean deliveries, and others showing no difference $(37,45,46)$.

When comparing elective to emergent or urgent C-section, a higher volume of cord blood has been found with elective $\mathrm{C}$-sections, although the quality of the cells appears to be improved in urgent or emergent C-sections. The higher volume collected in elective C-sections is thought to be primarily due to their scheduled nature, which allows for a more organized UCB collection process. Emergent or urgent $\mathrm{C}$-sections lead to a higher TNC and CD34+ cells collected vs. scheduled C-sections $(42,50)$, which may be due to the fetal distress that frequently leads to the emergent or urgent procedures.

One of the most important lessons that can be derived from the research on delivery mode and UCB collection is that the environment surrounding delivery is not binary. Limiting the description of the delivery experience to vaginal vs. cesarean delivery ignores many critical factors in UCB collection, including timing of cord clamping, placental manipulation, duration of labor, and the amount of fetal distress. With that in mind, it is worth noting that many of the studies cited here were performed before the widespread adoption of DCC practices.

\section{THEORIZED MECHANISMS OF ACTION OF UCB IN BRAIN INJURY}

In addition to selecting the most effective stem cell populations for transplantation, optimizing the clinical efficacy of stem cell transplantation in neurologic injury will require a thorough understanding of the underlying neuroprotective mechanisms of action of the UCB stem cells. With improved knowledge regarding stem cell signaling, we may also develop the ability to further select UCB cell subpopulations or alter cell expression to provide increased transplantation efficacy. With this in mind, the following section will discuss some key components of the stem cell secretome.

To date, there remains considerable debate regarding the mechanisms of action of the MSC's neuroprotective and neuroregenerative properties. Although stem cells have been shown to possess the capacity to develop into adult neurons, oligodendrocytes, and astrocytes in vitro and in vivo (51), it is now widely believed that they primarily function more distally through autocrine and paracrine mechanisms by the release of cytokines, growth factors, and other signaling molecules. This theory is supported by studies demonstrating decreased brain injury after treatment with cell-free MSC-conditioned media that is known to contain factors such as insulin-like growth factor-1 (IGF-1) and nerve growth factor (NGF) $(52,53)$.

MSC transplantation results in the upregulation of two primary classes of molecules that are known to affect brain growth and development (Figure 3). The first class consists of the neurotrophins, which promote the survival and differentiation of neural stem cells and neuroprogenitor cells, and include brain-derived neurotrophic factor (BDNF) and glial cell-derived neurotrophic factor. BDNF and glial cell-derived neurotrophic factor are secreted by human BM-derived MSCs, and may be primary components of the neuroprotective effects of MSCs, as blocking their activity significantly attenuates the neuroprotective effect of

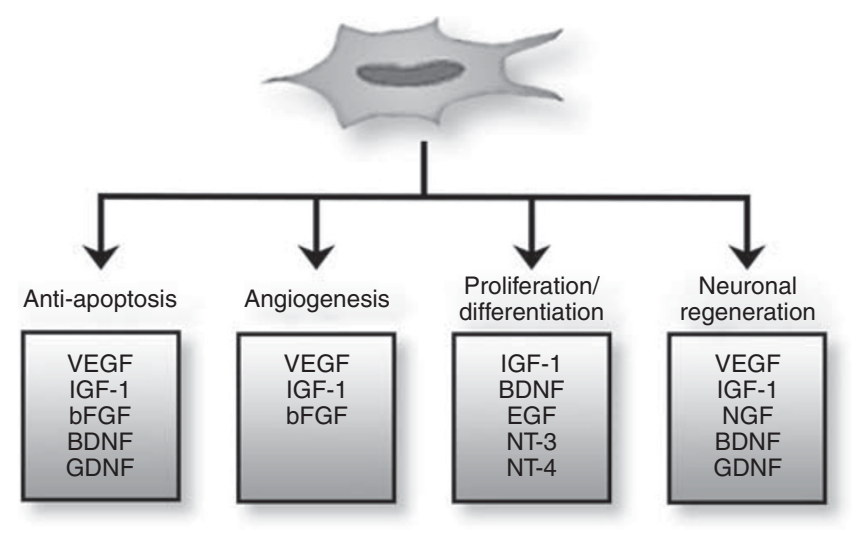

Figure 3. Examples of the neuroprotective actions of the stem cell secretome. bFGF, basic fibroblast growth factor; BDNF, brain-derived neurotrophic factor; EGF, epidermal growth factor; GDNF, glial cellderived neurotrophic factor; IGF-1, insulin-like growth factor-1; NGF, nerve growth factor; NT-3, neurotrophin-3; NT-4, neurotrophin-4; VEGF, vascular endothelial growth factor. 


\section{Review | Maillacheruvu et al.}

stem cell-conditioned media $(54,55)$. In a neonatal rat model of HIE, intracerebral BDNF administration significantly decreases caspase-3 activation and tissue loss (56,57). Similarly, NGF is a neurotrophin that aids in the survival and differentiation of neurons and has been shown to be expressed at higher levels after MSC transplantation, both by the host tissue as well as the grafted MSCs (58-60). The only published report of NGF administration in human neonates demonstrated clinical improvement in electroencephalography, cerebral perfusion, and a marker neurogenesis in two infants who received intraventricular injections of NGF after hypoxic-ischemic injury (61).

The second class of molecules upregulated by MSC transplantation consists of the neuroprotective cytokines, including IGF-1. IGF-1 is a growth factor that has been detected in BM MSC-conditioned medium (62), and has been demonstrated to improve BM MSC survival and proliferation during early differentiation $(63,64)$. Inhibiting IGF-1 activity in a model of cerebral infarction resulted in a significant decrease in neural progenitor proliferation (65). In a neonatal animal model, intranasal or intracranial IGF-1 increased progenitor cell proliferation in the subventricular zone, attenuated brain injury, and improved neurobehavioral performance when administered after induced hypoxicischemic brain injury $(66,67)$.

Understanding these molecular factors and their effects may allow investigators in the future to modify stem cells before transplantation to up- or downregulate certain factors to optimize therapeutic effects.

\section{STATUS OF CLINICAL ADMINISTRATION OF UCB FOR PEDIATRIC BRAIN INJURY}

Some of the earliest clinical studies of stem cell administration for nonhematological disorders were performed on infants and children with metabolic disorders. A study in 2004 demonstrated feasibility and favorable outcomes after UCB transplantation in children with Hurler's disease (68), which was followed shortly by two additional studies detailing the administration of UCB to infants with Krabbe's disease (69) and several other lysosomal and peroxisomal storage disorders (70). With these studies demonstrating feasibility and relative safety, and the mounting preclinical literature, investigators have begun to evaluate stem cell administration for a wide range of neonatal and pediatric brain disorders.

Although they are some of the best-studied acute neurological diseases of childhood in the preclinical literature, HIE and stroke have only just begun to produce early clinical trials of stem cell transplantation. One clinical study has been performed in infants after HIE which demonstrated clinical feasibility as well as early positive benefits with improved developmental outcomes at 1 year of life (4). The same investigators are currently undertaking a phase II randomized controlled trial of UCB in HIE, using survival at 1 year and Bayley Scales of Infant Development $\geq 85$ as their primary outcome measures. There may be a surge of data in this field in the near future, as there are currently seven other clinical trials registered with Clinicaltrials.gov, two of which are randomized controlled trials.

Many of the other perinatal and neonatal brain injuries have even less clinical data to support UCB transplantation. A recent study described a case report of UCB administered 4 years after a perinatal stroke that resulted in significant improvement in the subject's motor function (71). No clinical trials in perinatal stroke have been performed, however, and only one is currently ongoing, but has suspended recruitment. Similarly, phase I and phase II trials are in progress to evaluate the benefits of a commercial stem cell product on intraventricular hemorrhage in preterm infants, but no results have been published. Last, feasibility and safety of UCB transplantation have been assessed in congenital hydrocephalus (72), but no studies have evaluated outcomes in this population.

Chronic pediatric brain disorders generate added questions regarding timing of $\mathrm{UCB}$ administration and appropriate number of repeated transplantations. Nevertheless, several clinical trials have begun to evaluate the use of UCB stem cells in chronic pediatric disorders such as cerebral palsy (3,73-81). Although several of these studies have included only a few patients, as a whole they contain over 600 subjects and demonstrate feasibility and safety of UCB administration in this population. Thorough evaluation of efficacy and the development of a standardized administration protocol for these patients, however, is still needed, and will hopefully be provided by several of the clinical trials that are currently in progress, including two here in the United States.

\section{LIMITATIONS TO THE CLINICAL USE OF STEM CELLS FOR PEDIATRIC BRAIN INJURY}

One question that must be answered before clinical implementation of stem cells for brain injury is the ideal route of administration. Several different routes have been trialed in preclinical studies using stem cell transplantation for brain injury. These include intraventricular, intraperitoneal, intravenous, intrathecal, intranasal, and intraparenchymal injections into the cerebral cortex (82-89). One study examined the difference between intravenous and intraparenchymal transplantation of UCB MSCs in treating cerebral hypoxic injury. They found that intravenous administration resulted in better long-term outcomes than intraparenchymal, although both had better developmental recovery than nontransplanted animals (82). Similarly, a study using Wharton's jelly stem cells in a rat model of HIE demonstrated that intravenous administration resulted in improved behavioral outcomes and decreased gliosis in the areas of injury. Intraperitoneal transplantation in the same population exhibited less improvement compared with intravenous (86). Intravenous administration therefore appears to be the least invasive and most effective mode of administration. Recently, however, studies have begun to assess the efficacy of intranasal administration, which has shown to be safe and effective in neonatal brain injury (84,90-92). No comparison studies between intravenous and intranasal administration in 


\section{Umbilical cord cells and the injured brain ReVieW}

pediatric brain injury have been reported. For now, clinical trials have been limited to intravenous, intraventricular, and intrathecal administration, and have not yet evaluated the intranasal route $(4,81,83)$.

As with all therapies, it is important to thoroughly evaluate potential short- and long-term adverse effects of administering neuroprotective doses of stem cells or stem cell factors. One of the main concerns of stem cell transplantation is the development of neoplasms. It is believed that the tumorigenicity of UCB cells is less than that of embryonic or induced pluripotent cells (93), and a meta-analysis of over 1,000 subjects showed that intravenous or intra-arterial administration showed no association with the development of malignancy (94). Stem cell molecular factors alone may also pose risk, as BDNF and long-term IGF-1 therapy may be epileptogenic $(95,96)$.

\section{CONCLUSION}

While stem cell therapy for neonatal and pediatric brain injury has demonstrated significant promise, many details of the therapy remain under investigation. For instance, to select ideal UCB units for therapy, numerous perinatal factors should be considered, most significantly the birth weight and gestational age at the time of birth. Future research should focus on the effects of perinatal factors on UCB stem cell function, as opposed to simply cell type and number, and attempt to better define the effects of race and ethnicity by obtaining more detailed demographic data or using genetic analyses. Early results from studies using stem cell-secreted neurotrophic and growth factors have shown similar success in attenuating brain injury; however, the short- and long-term adverse effects of these therapies have not yet been well evaluated.

\section{STATEMENT OF FINANCIAL SUPPORT}

No financial assistance was received in support of this study. We have no financial ties to any products described in the study.

Disclosure: The authors declare no conflict of interest.

\section{REFERENCES}

1. Lu D, Sanberg PR, Mahmood A, et al. Intravenous administration of human umbilical cord blood reduces neurological deficit in the rat after traumatic brain injury. Cell Transplant 2002;11:275-81.

2. Ahn SY, Chang YS, Park WS. Stem cells for neonatal brain disorders. Neonatology 2016;109:377-83.

3. Kang M, Min K, Jang J, et al. Involvement of immune responses in the efficacy of cord blood cell therapy for cerebral palsy. Stem Cells Dev 2015;24:2259-68.

4. Cotten CM, Murtha AP, Goldberg RN, et al. Feasibility of autologous cord blood cells for infants with hypoxic-ischemic encephalopathy. J Pediatr 2014;164:973-9 e971.

5. Lee M, Jeong SY, Ha J, et al. Low immunogenicity of allogeneic human umbilical cord blood-derived mesenchymal stem cells in vitro and in vivo. Biochem Biophys Res Commun 2014;446:983-9.

6. Han P, Hodge G, Story C, Xu X. Phenotypic analysis of functional T-lymphocyte subtypes and natural killer cells in human cord blood: relevance to umbilical cord blood transplantation. Br J Haematol 1995;89: $733-40$.
7. Taghizadeh RR, Pollok KE, Betancur M, et al. Wharton's jelly derived mesenchymal stem cells: regenerative medicine beyond umbilical cord blood. Placenta 2011;32:S339.

8. Karahuseyinoglu S, Cinar O, Kilic E, et al. Biology of the stem cells in human umbilical cord stroma: in situ and in vitro surveys. Stem Cells 2007;25:319-31.

9. Fong CY, Richards M, Manasi N, Biswas A, Bongso A. Comparative growth behaviour and characterization of stem cells from human Wharton's jelly. Reprod Biomed Online 2007;15:708-18.

10. Soncini M, Vertua E, Gibelli L, et al. Isolation and characterization of mesenchymal cells from human fetal membranes. J Tissue Eng Regen Med 2007;1:296-305.

11. Dominici M, Le Blanc K, Mueller I, et al. Minimal criteria for defining multipotent mesenchymal stromal cells. The International Society for Cellular Therapy Position Statement. Cytotherapy 2006;8:315-7.

12. Divya MS, Roshin GE, Divya TS, et al. Umbilical cord blood-derived mesenchymal stem cells consist of a unique population of progenitors coexpressing mesenchymal stem cell and neuronal markers capable of instantaneous neuronal differentiation. Stem Cell Res Ther 2012;3:57.

13. Goodwin HS, Bicknese AR, Chien SN, et al. Multilineage differentiation activity by cells isolated from umbilical cord blood: expression of bone, fat, and neural markers. Biol Blood Marrow Transplant 2001;7:581-8.

14. Meier C, Middelanis J, Wasielewski B, et al. Spastic paresis after perinatal brain damage in rats is reduced by human cord blood mononuclear cells. Pediatr Res 2006;59:244-9.

15. Xia G, Hong X, Chen X, Lan F, Zhang G, Liao L. Intracerebral transplantation of mesenchymal stem cells derived from human umbilical cord blood alleviates hypoxic ischemic brain injury in rat neonates. J Perinat Med 2010;38:215-21.

16. Pimentel-Coelho PM, Magalhaes ES, Lopes LM, deAzevedo LC, Santiago MF, Mendez-Otero R. Human cord blood transplantation in a neonatal rat model of hypoxic-ischemic brain damage: functional outcome related to neuroprotection in the striatum. Stem Cells Dev 2010;19:351-8.

17. Mareschi K, Biasin E, Piacibello W, Aglietta M, Madon E, Fagioli F. Isolation of human mesenchymal stem cells: bone marrow versus umbilical cord blood. Haematologica 2001;86:1099-100.

18. Secco M, Zucconi E, Vieira NM, et al. Multipotent stem cells from umbilical cord: cord is richer than blood! Stem Cells 2008;26:146-50.

19. Al-Qahtani R, Al-Hedythi S, Arab S, Aljuhani A, Jawdat D. Factor predicting total nucleated cell counts in cord blood units. Transfusion 2016;56:2352-4.

20. Bieback K, Kern S, Kluter H, Eichler H. Critical parameters for the isolation of mesenchymal stem cells from umbilical cord blood. Stem Cells 2004;22:625-34.

21. Page KM, Mendizabal A, Betz-Stablein B, et al. Optimizing donor selection for public cord blood banking: influence of maternal, infant, and collection characteristics on cord blood unit quality. Transfusion 2014;54:340-52.

22. Allan DS, Scrivens N, Lawless T, et al. Delayed clamping of the umbilical cord after delivery and implications for public cord blood banking. Transfusion 2016;56:662-5.

23. Frandberg S, Waldner B, Konar J, Rydberg L, Fasth A, Holgersson J. High quality cord blood banking is feasible with delayed clamping practices. The eight-year experience and current status of the national Swedish Cord Blood Bank. Cell Tissue Bank 2016;17:439-48.

24. Sanberg PR, Divers R, Mehindru A, Mehindru A, Borlongan CV. Delayed umbilical cord blood clamping first line of defense against neonatal and age-related disorders. Wulfenia 2014;21:243-9.

25. Lawton C, Acosta S, Watson N, et al. Enhancing endogenous stem cells in the newborn via delayed umbilical cord clamping. Neural Regen Res 2015;10:1359-62.

26. Tolosa JN, Park DH, Eve DJ, Klasko SK, Borlongan CV, Sanberg PR. Mankind's first natural stem cell transplant. J Cell Mol Med 2010;14: 488-95.

27. Committee on Obstetric Practice. Committee Opinion No. 684: Delayed Umbilical Cord Clamping After Birth. Obstet Gynecol 2017;129:e5-e10. 
28. Katheria AC, Truong G, Cousins L, Oshiro B, Finer NN. Umbilical cord milking versus delayed cord clamping in preterm infants. Pediatrics 2015;136:61-9.

29. Rabe H, Jewison A, Alvarez RF, et al. Milking compared with delayed cord clamping to increase placental transfusion in preterm neonates: a randomized controlled trial. Obstet Gynecol 2011;117:205-11.

30. Huang C, Chen D, Wei H, et al. Hypoxia-induced therapeutic neovascularization in a mouse model of an ischemic limb using cell aggregates composed of HUVECs and cbMSCs. Biomaterials 2013;34: 9441-50.

31. Lavrentieva A, Majore I, Kasper C, Hass R. Effects of hypoxic culture conditions on umbilical cord-derived human mesenchymal stem cells. Cell Commun Signal 2010;8:18.

32. Blue E, DiGiuseppe R, Derr-Yellin E, et al. Gestational diabetes induces alterations in the function of neonatal endothelial colony forming cells. Pediatr Res 2014;75:266-72.

33. Dincer UD. Fetal exposure to a diabetic intrauterine environment resulted in a failure of cord blood endothelial progenitor cell adaptation against chronic hypoxia. Stem Cells Cloning 2015;8:1-14.

34. Dincer U. Moderate hypoxia exhibits increased endothelial progenitor vessel-forming ability however gestational diabetes caused to impede compensatory defense reaction. Int J Stem Cells 2016;9:152-62.

35. Munoz-Hernandez R, Miranda ML, Stiefel P, et al. Decreased level of cord blood circulating endothelial colony-forming cells in preeclampsia. Hypertension 2014;64:165-71.

36. Wahid FS, Nasaruddin MZ, Idris MR, Tusimin M, Tumian NR, Mahdy ZA. Effects of preeclampsia on the yield of hematopoietic stem cells obtained from umbilical cord blood at delivery. J Obstet Gynaecol Res 2012;38:490-7.

37. Al-Sweedan SA, Musalam L, Obeidat B. Factors predicting the hematopoietic stem cells content of the umbilical cord blood. Transfus Apher Sci 2013;48:247-52.

38. Ballen KK, Wilson M, Wuu J, et al. Bigger is better: maternal and neonatal predictors of hematopoietic potential of umbilical cord blood units. Bone Marrow Transplant 2001;27:7-14.

39. Manegold-Brauer G, Borner B, Bucher C, et al. A prenatal prediction model for total nucleated cell count increases the efficacy of umbilical cord blood banking. Transfusion 2014;54:2946-52.

40. Jones J, Stevens CE, Rubinstein P, Robertazzi RR, Kerr A, Cabbad MF. Obstetric predictors of placental/umbilical cord blood volume for transplantation. Am J Obstet Gynecol 2003;188:503-9.

41. Nakagawa R, Watanabe T, Kawano Y, et al. Analysis of maternal and neonatal factors that influence the nucleated and CD34+ cell yield for cord blood banking. Transfusion 2004;44:262-7.

42. Santos SV, Barros SM, Santos MS, et al. Predictors of high-quality cord blood units. Transfusion 2016;56:2030-6.

43. Reham AQ, Sara AH, Suha A, Amal A, Dunia J. Factor predicting total nucleated cell counts in cord blood units. Transfusion 2016;56:2352-4.

44. Wyrsch A, Carbonare V, Jansen W, et al. Umbilical cord blood from preterm human fetuses is rich in committed and primitive hematopoietic progenitors with high proliferative and self-renewal capacity. Exp Hematol 1999;27:1338-45.

45. Cairo MS, Wagner EL, Fraser J, et al. Characterization of banked umbilical cord blood hematopoietic progenitor cells and lymphocyte subsets and correlation with ethnicity, birth weight, sex, and type of delivery: a Cord Blood Transplantation (COBLT) Study Report. Transfusion 2005;45:856-66.

46. Aroviita P, Teramo K, Hiilesmaa V, Kekomäki R. Cord blood hematopoietic progenitor cell concentration and infant sex. Transfusion 2005;45:613-21.

47. Bijou F, Ivanovic Z, Fizet D, Dazey B, Boiron JM. Neonatal sex and weight influence CD34+ cell concentration in umbilical cord blood but not stromal cell-derived factor 1-3' A polymorphism. Cytotherapy 2015;17:68-72.
48. Yang H, Loutfy MR, Mayerhofer S, Shuen P. Factors affecting banking quality of umbilical cord blood for transplantation. Transfusion 2011;51: 284-92.

49. Mancinelli F, Tamburini A, Spagnoli A, et al. Optimizing umbilical cord blood collection: impact of obstetric factors versus quality of cord blood units. Transplant Proc 2006;38:1174-6.

50. Gwendolin M, Sandrine MM, Andre T, Doris P, Wolfgang H, Carolyn T. Cesarean section due to fetal distress increases the number of stem cells in umbilical cord blood. Transfusion 2008;48:871-6.

51. Song HJ, Stevens CF, Gage FH. Neural stem cells from adult hippocampus develop essential properties of functional CNS neurons. Nat Neurosci 2002;5:438-45.

52. Chen $\mathrm{X}$, Li Y, Wang $\mathrm{L}$, et al. Ischemic rat brain extracts induce human marrow stromal cell growth factor production. Neuropathology 2002;22: 275-9.

53. Wei X, Du Z, Zhao L, et al. IFATS Collection: the conditioned media of adipose stromal cells protect against hypoxia-ischemia-induced brain damage in neonatal rats. Stem Cells 2009;27:478-88.

54. Wilkins A, Kemp K, Ginty M, Hares K, Mallam E, Scolding N. Human bone marrow-derived mesenchymal stem cells secrete brain-derived neurotrophic factor which promotes neuronal survival in vitro. Stem Cell Res 2009;3:63-70.

55. Whone AL, Kemp K, Sun M, Wilkins A, Scolding NJ. Human bone marrow mesenchymal stem cells protect catecholaminergic and serotonergic neuronal perikarya and transporter function from oxidative stress by the secretion of glial-derived neurotrophic factor. Brain Res 2012;1431:86-96.

56. Cheng Y, Gidday JM, Yan Q, Shah AR, Holtzman DM. Marked agedependent neuroprotection by brain-derived neurotrophic factor against neonatal hypoxic-ischemic brain injury. Ann Neurol 1997;41:521-9.

57. Han BH, D'Costa A, Back SA, et al. BDNF blocks caspase-3 activation in neonatal hypoxia-ischemia. Neurobiol Dis 2000;7:38-53.

58. Lu P, Jones LL, Snyder EY, Tuszynski MH. Neural stem cells constitutively secrete neurotrophic factors and promote extensive host axonal growth after spinal cord injury. Exp Neurol 2003;181:115-29.

59. Chen J, Zheng S, Huang H, et al. Mesenchymal stem cells enhanced cardiac nerve sprouting via nerve growth factor in a rat model of myocardial infarction. Curr Pharm Des 2014;20:2023-9.

60. Holtzman DM, Sheldon RA, Jaffe W, Cheng Y, Ferriero DM. Nerve growth factor protects the neonatal brain against hypoxicischemic injury. Ann Neurol 1996;39:114-22.

61. Chiaretti A, Antonelli A, Genovese O, et al. Intraventricular nerve growth factor infusion improves cerebral blood flow and stimulates doublecortin expression in two infants with hypoxic-ischemic brain injury. Neurol Res 2008;30:223-8.

62. Tögel F, Weiss K, Yang Y, Hu Z, Zhang P, Westenfelder C. Vasculotropic, paracrine actions of infused mesenchymal stem cells are important to the recovery from acute kidney injury. Am J Physiol 2007;292:F1626.

63. Huat TJ, Khan AA, Pati S, Mustafa Z, Abdullah JM, Jaafar H. IGF-1 enhances cell proliferation and survival during early differentiation of mesenchymal stem cells to neural progenitor-like cells. BMC Neurosci 2014;15:91.

64. Dempsey RJ, Sailor KA, Bowen KK, Tureyen K, Vemuganti R. Strokeinduced progenitor cell proliferation in adult spontaneously hypertensive rat brain: effect of exogenous IGF-1 and GDNF. J Neurochem 2003;87: 586-97.

65. Yan YP, Sailor KA, Vemuganti R, Dempsey RJ. Insulin-like growth factor-1 is an endogenous mediator of focal ischemia-induced neural progenitor proliferation. Eur J Neurosci 2006;24:45-54.

66. Lin S, Fan LW, Pang Y, Rhodes PG, Mitchell HJ, Cai Z. IGF-1 protects oligodendrocyte progenitor cells and improves neurological functions following cerebral hypoxia-ischemia in the neonatal rat. Brain Res 2005; 1063:15-26.

67. Lin S, Fan LW, Rhodes PG, Cai Z. Intranasal administration of IGF-1 attenuates hypoxic-ischemic brain injury in neonatal rats. Exp Neurol 2009;217:361-70. 


\section{Umbilical cord cells and the injured brain Review}

68. Staba SL, Escolar ML, Poe M, et al. Cord-blood transplants from unrelated donors in patients with Hurler's syndrome. N Engl J Med 2004;350:1960-9.

69. Escolar ML, Poe MD, Provenzale JM, et al. Transplantation of umbilicalcord blood in babies with infantile Krabbe's disease. N Engl J Med 2005;352:2069-81.

70. Martin PL, Carter SL, Kernan NA, et al. Results of the cord blood transplantation study (COBLT): outcomes of unrelated donor umbilical cord blood transplantation in pediatric patients with lysosomal and peroxisomal storage diseases. Biol Blood Marrow Transplant 2006;12: $184-94$.

71. Jensen A, Hamelmann E. First autologous cord blood therapy for pediatric ischemic stroke and cerebral palsy caused by cephalic molding during birth: individual treatment with mononuclear cells. Case Rep Transplant 2016;2016:1717426.

72. Sun JM, Grant GA, McLaughlin C, et al. Repeated autologous umbilical cord blood infusions are feasible and had no acute safety issues in young babies with congenital hydrocephalus. Pediatr Res 2015;78:712-6.

73. Min K, Song J, Kang JY, et al. Umbilical cord blood therapy potentiated with erythropoietin for children with cerebral palsy: a double-blind, randomized, placebo-controlled trial. Stem Cells 2013;31:581-91.

74. Romanov YA, Tarakanov OP, Radaev SM, et al. Human allogeneic $\mathrm{AB} 0 / \mathrm{Rh}$-identical umbilical cord blood cells in the treatment of juvenile patients with cerebral palsy. Cytotherapy 2015;17:969-78.

75. Lee $\mathrm{YH}$, Choi KV, Moon JH, et al. Safety and feasibility of countering neurological impairment by intravenous administration of autologous cord blood in cerebral palsy. J Transl Med 2012;10:58.

76. Sun J, Allison J, McLaughlin C, et al. Differences in quality between privately and publicly banked umbilical cord blood units: a pilot study of autologous cord blood infusion in children with acquired neurologic disorders. Transfusion 2010;50:1980-7.

77. Yang WZ, Zhang Y, Wu F, et al. Safety evaluation of allogeneic umbilical cord blood mononuclear cell therapy for degenerative conditions. J Transl Med 2010;8:75.

78. Feng M, Lu A, Gao H, et al. Safety of allogeneic umbilical cord blood stem cells therapy in patients with severe cerebral palsy: a retrospective study. Stem Cells Int 2015;2015:325652.

79. Wang $\mathrm{X}, \mathrm{Hu} \mathrm{H}, \mathrm{Hua} \mathrm{R}$, et al. Effect of umbilical cord mesenchymal stromal cells on motor functions of identical twins with cerebral palsy: pilot study on the correlation of efficacy and hereditary factors. Cytotherapy 2015;17:224-31.

80. Miao X, Wu X, Shi W. Umbilical cord mesenchymal stem cells in neurological disorders: a clinical study. Indian J Biochem Biophys 2015;52:140-6.

81. Abi Chahine NH, Wehbe TW, Hilal RA, Zoghbi VV, Melki AE, Bou Habib EB. Treatment of cerebral palsy with stem cells: a report of 17 cases. Int J Stem Cells 2016;9:90-5.
82. Willing AE, Lixian J, Milliken $\mathrm{M}$, et al. Intravenous versus intrastriatal cord blood administration in a rodent model of stroke. J Neurosci Res 2003;73:296-307.

83. Ahn SY, Chang YS, Sung DK, et al. Mesenchymal stem cells prevent hydrocephalus after severe intraventricular hemorrhage. Stroke 2013;44: 497-504.

84. van Velthoven CTJ, Kavelaars A, van Bel F, Heijnen CJ. Nasal administration of stem cells: a promising novel route to treat neonatal ischemic brain damage. Pediatr Res 2010;68:419-22.

85. Chang YS, Oh W, Choi SJ, et al. Human umbilical cord blood-derived mesenchymal stem cells attenuate hyperoxia-induced lung injury in neonatal rats. Cell Transplant 2009;18:869-86.

86. Zhang X, Zhang Q, Li W, et al. Therapeutic effect of human umbilical cord mesenchymal stem cells on neonatal rat hypoxic-ischemic encephalopathy. J Neurosci Res 2014;92:35-45.

87. Wu KJ, Yu SJ, Chiang CW, et al. Transplantation of human placentaderived multipotent stem cells reduces ischemic brain injury in adult rats. Cell Transplant 2015;24:459-70.

88. Hocum Stone LL, Xiao F, Rotschafer J, et al. Amelioration of ischemic brain injury in rats with human umbilical cord blood stem cells: mechanisms of action. Cell Transplant 2016;25:1473-88.

89. Fang B, Wang H, Sun XJ, et al. Intrathecal transplantation of bone marrow stromal cells attenuates blood-spinal cord barrier disruption induced by spinal cord ischemia-reperfusion injury in rabbits. J Vasc Surg 2013;58:1043-52.

90. Donega V, van Velthoven CT, Nijboer CH, et al. Intranasal mesenchymal stem cell treatment for neonatal brain damage: long-term cognitive and sensorimotor improvement. PLoS ONE 2013;8:e51253.

91. Donega V, Nijboer CH, Braccioli L, et al. Intranasal administration of human MSC for ischemic brain injury in the mouse: in vitro and in vivo neuroregenerative functions. PLoS ONE 2014;9:e112339.

92. Donega V, Nijboer CH, van Velthoven CT, et al. Assessment of long-term safety and efficacy of intranasal mesenchymal stem cell treatment for neonatal brain injury in the mouse. Pediatr Res 2015;78:520-6.

93. Park SJ, Kim HJ, Kim W, et al. Tumorigenicity evaluation of umbilical cord blood-derived mesenchymal stem cells. Toxicol Res 2016;32:251-8.

94. Lalu MM, McIntyre L, Pugliese C, et al. Safety of cell therapy with mesenchymal stromal cells (SafeCell): a systematic review and metaanalysis of clinical trials. PLoS ONE 2012;7:e47559.

95. Song Y, Pimentel C, Walters K, et al. Neuroprotective levels of IGF-1 exacerbate epileptogenesis after brain injury. Sci Rep 2016;6:32095.

96. Scharfman HE, Goodman JH, Sollas AL, Croll SD. Spontaneous limbic seizures after intrahippocampal infusion of brain-derived neurotrophic factor. Exp Neurol 2002;174:201-14.

97. Uthman E. The umbilical cord. Flickr 2009. https://www.flickr.com/ photos/euthman/3276268079. 